\title{
Regional network analysis of GPS data in the area of Kanto, central Japan
}

\author{
K. Vijaykumar and K. Miyashita \\ Graduate School of Science and Engineering, Ibaraki University, Mito 310-8512, Japan
}

(Received January 26, 2000; Revised June 19, 2000; Accepted August 15, 2000)

\begin{abstract}
In order to monitor the crustal deformation in the area of eastern Kanto, central Japan, Ibaraki University has started the high precision geodetic studies with GPS measurements in collaboration with Geographical Survey Institute of Japan (GSI). The first measurements were taken in 1991 with a small network of 8 sites. Four more sites were included since 1998 for a better coverage in the region. In the present study, we analyzed the data collected since 1998 by using the GAMIT/GLOBK software for estimating the displacement vectors in the ITRF96 reference frame. The data from the continuous GPS network of GSI and the GPS sites of IGS close to the studied region (Tsukuba and Usuda) were included for obtaining the primary solutions. The initial results by constraining the co-ordinates of the IGS stations nearest to the network confirmed the repeatability of the baseline components. The results presented here were obtained by assuming the 4 fiducial stations around the network, namely, Irkutsks (IRKT), Fairbanks (FAIR), Sheshan (SHAO) and Dededo (GUAM). The average uncertainties of the horizontal velocities from three consecutive campaigns, each separated by six months, were estimated to be $\pm 4.6 \mathrm{~mm} / \mathrm{yr}$ and $\pm 3.5 \mathrm{~mm} / \mathrm{yr}$ for the northward and eastward components, respectively.
\end{abstract}

\section{Introduction}

As highlighted in Fig. 1, the eastern Kanto region in central Japan is located near the boundaries between the Philippine Sea (PHS) and North American (NAM) plates and between the Pacific (PAC) and NAM plates. Studies on the crustal deformation in this region have been of special interest because disastrous large earthquakes, e.g., the 1923 Kanto earthquake (M7.9), have occurred repeatedly along the Sagami trough, the plate boundary between the PHS and NAM plates.

It is a well-established idea that geodetic data for monitoring the deformation in regional and global scales can be used for predicting a future large earthquake. In the last few years the high accuracy of space geodetic data from GPS has dominated over that of conventional geodetic data. The main aim of the present study is to analyze the regional GPS data on the ITRF96 reference frame by combining our data with the global GPS data and to estimate the velocity field in this region.

\section{GPS Observations and Data Processing}

The data used in the present analysis were collected during the period from October 1998 to October 1999. Each of the three campaigns (October 1998, March 1999 and October 1999) was split into two sessions corresponding to the observations in the northern and southern halves of the studied area in respective durations. Each campaign ranged from 12 hrs to 36 hrs depending on the securities of the observation sites. We collected the data from 12 re-observation sites of Ibaraki and Chiba Universities and from 35 continuous observation sites of GSI. Observations were conducted with

Copy right (C) The Society of Geomagnetism and Earth, Planetary and Space Sciences (SGEPSS); The Seismological Society of Japan; The Volcanological Society of Japan; The Geodetic Society of Japan; The Japanese Society for Planetary Sciences.
Trimble 4000SSi receivers at a sampling rate of 30 seconds and an elevation mask of $15^{\circ}$. To have a better fiducial control, data from 4-5 IGS stations around the regional network were used after checking the performance of the stations. The data at the IGS stations close to the network and the precise ephemerides distributed were downloaded from the anonymous ftp sites of either IGS or SOPAC.

The data processing was carried out in two steps which are as follows:

First we processed all the available data by using dual frequency and double differenced GPS phase observations together with precise ephemerides of IGS. The post-processing software used was GAMIT software, developed by Massachusetts Institute of Technology and University of California, San Diego (King and Bock, 1998). The data from each session i.e., of each Julian day was processed separately. However, due to the maximum limitation of 40 sites (memory limitation), each session was split into two subnetworks, which were combined later to have a single day solution.

Data from 4 fiducial stations of IGS around the regional network were used in the analysis to improve the ambiguity resolution by putting tight constraints ( 3 to $5 \mathrm{~mm}$ on the horizontal and 5 to $10 \mathrm{~mm}$ on the vertical) on these sites from their a priori coordinate values in the ITRF96 frame. The only criterion adopted in selecting the fiducial stations is the closest and well performing stations for the day of the analysis, although the ideal situation is to have these stations at about equidistances from the centroid of the regional network. All the local site co-ordinates were allowed to vary freely by way of very loose constraints (100 meters) to avoid singularity of the normal matrix (Dong et al., 1998). Although GAMIT produces two kinds of solutions based on phase ambiguity resolutions via bias-free and bias-fixed solutions, we 


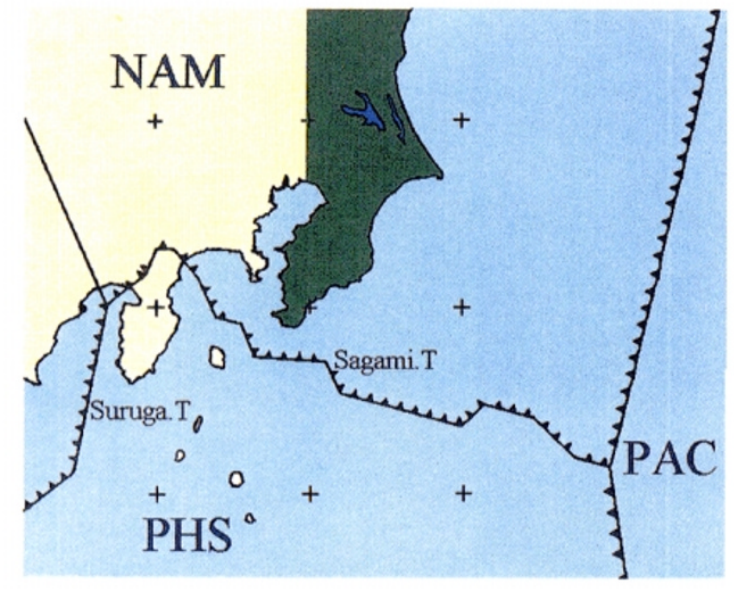

Fig. 1. Tectonic map in and around the present study region from the past geophysical studies. The green shade region represents the study region. All the plate boundaries are shown in black toothed lines. considered the bias-free solution. Thus, for each session we obtained a final solution (loose solution), basically a set of loosely constrained parameter estimates (station coordinates, orbital conditions and phase ambiguity parameters) and their covariance matrix. This "loose solution" (Feigl et al., 1993) allows us to define the reference frame at the time of combination.

In the second step, GLOBK, a global Kalman filtering software was used to combine the primary solution files with the global solution files of SOPAC for estimating the station positions and velocities. Thus, by including the global network solution we adopted the robust method for defining the reference frame which was done by way of minimizing the departure of station co-ordinates and velocities of the IGS stations around the area concerned (Herring, 1998). Essentially a 7-parameter Helmert-transformation (three rotations, three translations and scale) was carried out to translate the

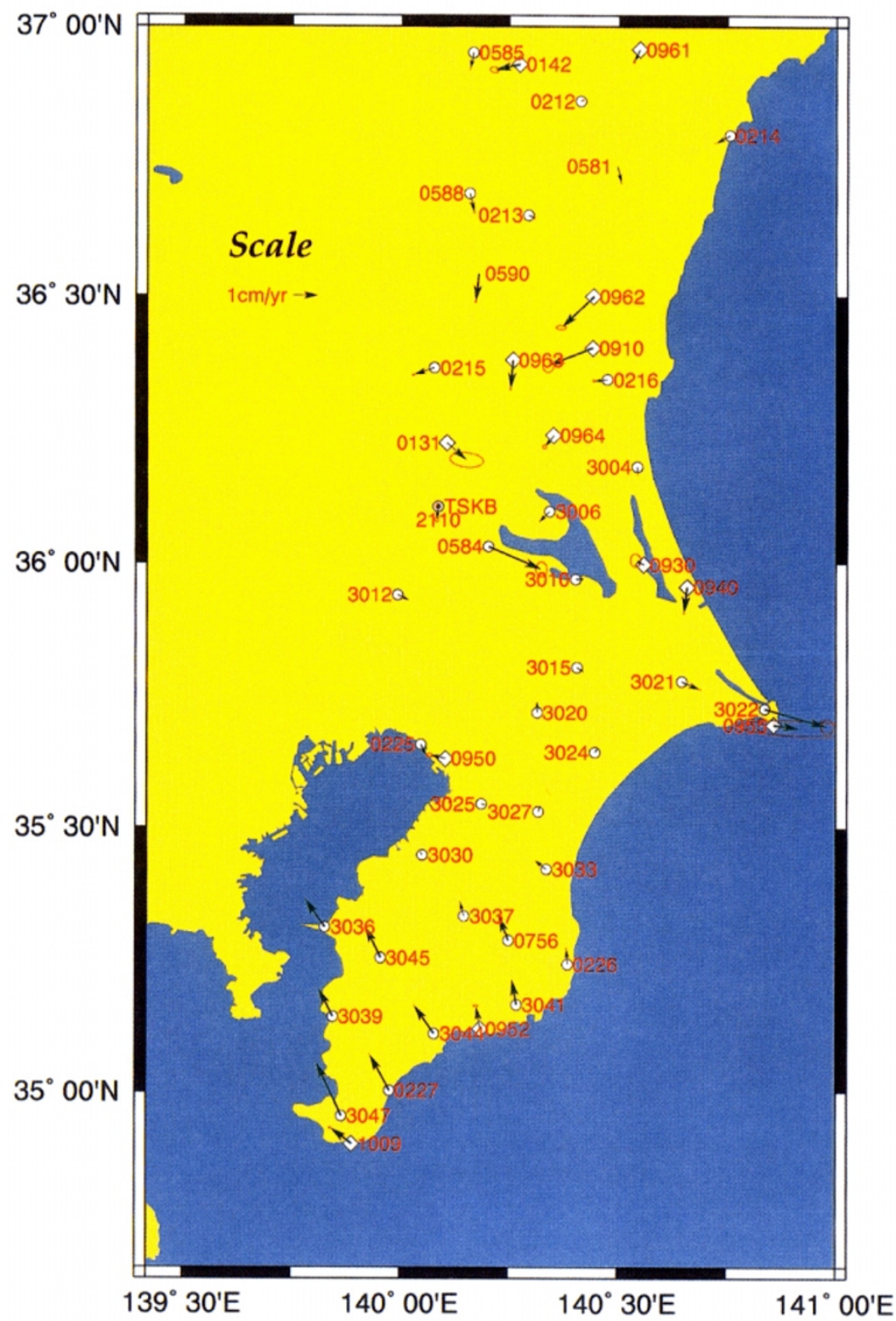

Fig. 2. Horizontal velocity vectors for all stations in the Eastern Kanto GPS Network relative to the GSI station 2110. The ellipses denote $95 \%$ confidence ellipses. University sites are represented with diamonds and GSI sites with circles. 

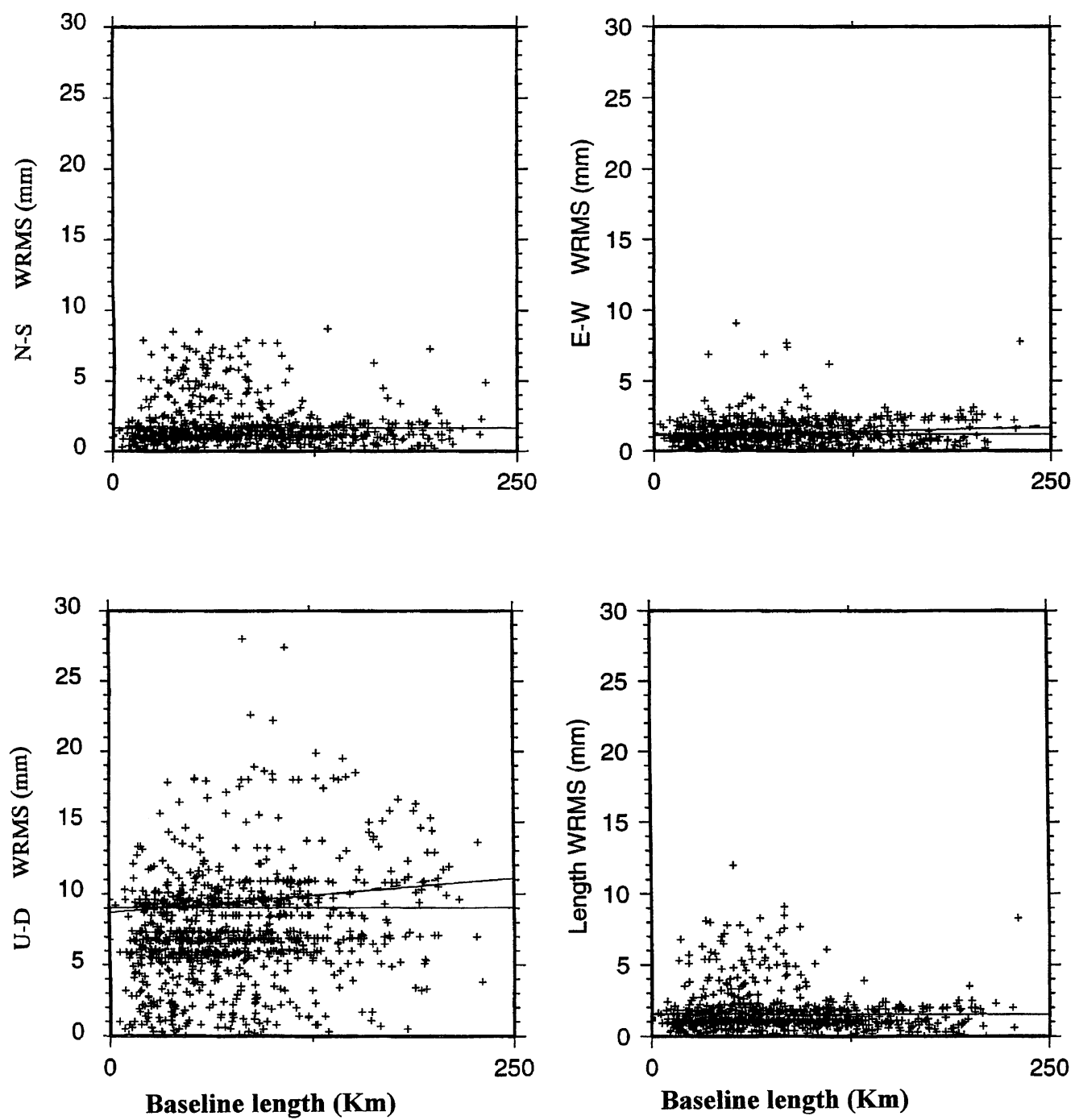

Fig. 3. Repeatability plot of the combined analysis for 1998-1999 data by constraining the Tsukuba IGS station. The weighted rms for all the three baseline components and the length are plotted against the baseline lengths.

regional network to global network. Technically this needs three stations and three observations to provide the minimum rank of the system. In the strict sense the GPS data is not free to translate, as the orbital dynamics are sensitive to the position and rate of the earth's center of mass. However, expecting the error due to poor modeling of the orbit we need to constrain the translation together with the rotation. The reference frame of coordinate system is defined by 13 of IGS core sites and elimination of the height component is made by setting a large value variance for the height component relative to those for the horizontal ones. We imposed a tight constraint $(1 \mathrm{~cm} / \mathrm{yr})$ on the horizontal velocities for some IGS sites, whereas we relaxed the constraints on velocities $(1 \mathrm{~m} / \mathrm{yr})$ and coordinates $(100 \mathrm{~m})$ for the local sites. The horizontal velocities of the stations obtained from at least two observations are shown in Fig. 2.

\section{Results}

The scatter plots of north, east, vertical and length components of the baseline vectors against the baseline lengths less than $250 \mathrm{~km}$ are shown in Fig. 3. The best fitting curve for the baseline repeatability shows that the errors are in the range of $1.65 \mathrm{~mm}+0.07 \mathrm{ppb}, 1.13 \mathrm{~mm}+2.2 \mathrm{ppb}$ and 8.69 $\mathrm{mm}+9.68 \mathrm{ppb}$ for the north, east and vertical components, respectively.

The velocities obtained in the second step give the rate changes of the station coordinates in the terrestrial reference frame. In order to study the deformation in the regional network it is considered essential to fix one of the stable sites. Assuming that the site 2110 is unaffected by unusual tectonic activities in the region, we estimated the relative horizontal velocities for the remaining sites (Fig. 2). All of the velocity vectors of the sites in the southern part of the eastern Kanto are directed towards the north-northwest, which agrees well with the earlier seismological studies (e.g., Ishibashi and Ishida, 1989; Ishida, 1992) showing an average rate of $2 \mathrm{~cm} / \mathrm{yr}$. In the northern part most of the sites show a westward movement except a few sites showing an eastward movement with an unduly high rate probably caused by the local movements. There has been considerable reduction of the outliers in the combined analysis of three campaigns over that of the first two campaigns. 


\section{Conclusions and Recommendations}

The general trend of the horizontal velocities could be inferred even with a short duration of GPS data and which is comparable with the estimates obtained by GSI for the region. Advantage to use global data in the estimation of station coordinates of the local network in the global coordinates has thus been verified in this study.

The most significant feature is that the vectors direct towards the northwest in the southern part of the Boso peninsula. This is considered to be the effect of the PHS plate subducting obliquely from the Sagami trough. Looking at the outliers on the university sites it is clear that more stringent steps should be taken in setting up the antennas at the sites during re-occupation to avoid the observational blunders.

To confirm the tectonic activity and for a better temporal coverage, it is considered essential to analyze data from longer observation period. Further, detailed strain analysis has to be carried out since there has been significant accumulation of the seismic moment in the Boso peninsula segment near the Sagami trough (Hashimoto and Jackson, 1993) where the last experienced earthquake was in 1703.

Acknowledgments. The authors are grateful to GSI for providing the GPS data from the CGPS network in the study region. We are also thankful to Dr. S. Shimada and Dr. P. Morgan for their helpful discussions during the analysis of the data with GAMIT/GLOBK.

\section{References}

Dong, D., T. A. Herring, and R. W. King, Estimating regional deformation from a combination of space and terrestrial geodetic data, J. Geod., 72 200-214, 1998.

Feigl, K. L., D. C. Agnew, Y. Bock, D. Dong, A. Donnellan, B. H. Hager, T. A. Herring, D. D. Jackson, T. H. Jordan, R. W. King, S. Larsen, K. M. Larson, M. H. Murray, Z. Shen, and F. H. Webb, Space geodetic measurement of crustal deformation in central and southern California, 1984-1992, J. Geophys. Res., 98, 21677-21712, 1993.

Hashimoto, M. and D. D. Jackson, Crustal deformation around Japan, J. Geophys. Res., 98, 16149-16166, 1993.

Herring, T. A., GLOBK: Global Kalman filter VLBI and GPS analysis program version 4.1, Massachusetts Institute of Technology, Cambridge, 1998.

King, R. W. and Y. Bock, Documentation for GAMIT GPS analysis software, Massachusetts Institute of Technology, and University of California at San Diego, 1998.

Ishibashi, K. and M. Ishida, Seismic activity in the south Fossa Magna, central Japan, Mod. Geol., 14, 19-33, 1989.

Ishida, M., Geometry and Relative motion of Philippine Sea Plate and Pacific Plate beneath Kanto-Tokai district, J. Geophys. Res., 97, 489-513, 1992.

K. Vijaykumar (e-mail: vijay@mcs.ipc.ibaraki.ac.jp) and K. Miyashita 